\title{
Experimental Investigation of the Flow Field in a Multistage Axial Flow Compressor
}

\author{
B. LAKSHMINARAYANA, N. SURYAVAMSHI and J. PRATO \\ Center for 6 as Turbines and Power, The Pennsylvania State University \\ R. MORITZ \\ Allison Engine Company
}

\begin{abstract}
The nature of the flow field in a three stage axial flow compressor, including a detailed survey at the exit of an embedded stator as well as the overall performance of the compressor is presented and interpreted in this paper. The measurements include area traverse of a miniature five hole probe $(1.07 \mathrm{~mm}$ dia) downstream of stator 2 , radial traverses of a miniature five hole probe at the inlet, downstream of stator 3 and at the exit of the compressor at various circumferential locations, area traverse of a low response thermocouple probe downstream of stator 2, radial traverses of a single sensor hot-wire probe at the inlet, and casing static pressure measurements at various circumferential and axial locations across the compressor at the peak efficiency operating point. Mean velocity, pressure and total temperature contours as well as secondary flow contours at the exit of the stator 2 are reported and interpreted. Secondary flow contours show the migration of fluid particles toward the core of the low pressure regions located near the suction side casing endwall corner.
\end{abstract}

Key Words: multistage axial compressor; experimental; flozv field; efficiency

\section{INTRODUCTION}

$\mathbf{T}$ The flow field in a multistage compressor includes large regions in which the endwall phenomena cause significant deviations from 2-D cascade performance. Viscous and clearance effects result in vigorous shearing with momentum and thermal transfer. In order to make good design choices, it is very desirable to know the extent and limits of these effects in order, for instance, to avoid choosing a design in which major endwall flow reversals are present near the design point. At the present time, allowance for such effects is typically made by including an artificial viscosity term into the flow model. The objective of the Penn State research is to provide a more scientific basis for the analysis of the multistage compressor flow field.

Most of the research hitherto carried out are confined to cascades or rotor alone configuration. Robinson [1992] provides a critical review of the recent advances in understanding the nature of the flow in multistage compressors. Recent aerodynamic studies include area traverses at the exit of stator 2 in a highly loaded compressor (Calvert et al. [1989]) and radial traverses at the exit of each blade row of a high speed multistage compressor (Falchetti [1992]). Stauter et al. [1991] provide details of unsteady flow field acquired from laser doppler velocimetry in a two-stage low speed axial flow compressor. Cherrett and Bryce [1992] conducted radial traverses of a high frequency pressure transducer behind the first three stages of a high speed multistage compressor operating at three different throttle settings. In spite of these advances, there is a lack of cohesive and detailed understanding of the flow features. Hence, there is a need for detailed understanding of the multistage compressor flow phenomena, especially the role played by temperature fluctuations, in a high speed multistage compressor. This is one of the objectives of the Penn State multistage compressor research program.

The multistage compressor recently installed at Penn State, was donated by Pratt and Whitney. The compressor runs at high speed (5410 RPM, $171 \mathrm{~m} / \mathrm{s}$ tip speed) with a temperature rise of $30^{\circ} \mathrm{C}$. This compressor falls in the middle range (in terms of blade speed and Mach number), between very high speed facilities tested in 
industries and the low speed facilities and has reasonable temperature rise. This is an ideal vehicle to investigate the features hitherto unexplored. Hence, the objectives of Penn State multistage compressor research program are to understand the nature of the unsteady three dimensional turbulent flow field upstream and downstream of an embedded blade row, and to gain a basic understanding of the spanwise mixing and unsteadiness arising from various blade rows. This should lead to improvement in design, performance, and analysis of multistage compressors.

This paper provides details of facility, instrumentation, overall performance and radial distribution of average properties (pressure, temperature, velocity and flow angles) at the exit of stator 2, rotor 3 and stator 3 , as well as the data on local flow field from an area traverse at the exit of stator 2. Further details on facility, instrumentation, data acquisition system, accuracy of measurement and the method of calculating overall performance variables can be found in Lakshminarayana et al. [1991].

\section{DESCRIPTION OF TEST FACILITY AND BLADING DETAILS}

The test compressor is a three stage axial flow compressor consisting of an inlet guide vane row and three stages of rotor and cantilevered stator blading with a rotating hub (Figure 1). The general specifications of the compressor are listed in Table 1. The compressor was donated to Penn State University by Pratt and Whitney Aircraft of United Technologies Corporation. The drive assembly comprises of the $372 \mathrm{KW}$ induction motor (with a frequency controlled Inverter) coupled to the compressor via a 1:1.67 ratio speed increaser gear box

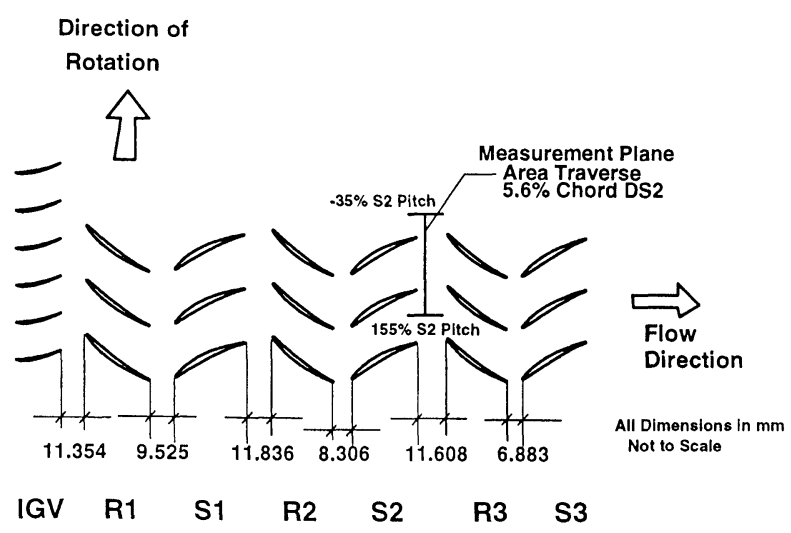

FIGURE 1 Schematic of Compressor Flow Path and Measurement Locations.
TABLE 1

General Specifications of Test Compressor

Number of Stages

Design Corrected Rotor speed

Design Corrected Mass Flow

Design Overall Total Pressure Ratio

Mass Averaged Peak Efficiency at $100 \%$

Corrected Speed (Torque Based)

Tip Diameter

Blade Tip Mach Number

Average Reynolds Number (Based on

Stator 3 chord length and axial velocity)

Average Hub-Tip Ratio

Diffusion factor (avg)

Average Flow Coefficient $\left(\mathrm{V}_{\mathrm{z}} / \mathrm{U}_{\mathrm{t}}\right)$

(Average for a Stage)

Reaction (avg)

Space Chord Ratio (avg)

Aspect Ratio (avg)

Average Rotor Tip Clearance (static)

Average Rotor Tip Clearance (dynamic)

Average Stator Hub Clearance (static)
3

$5410 \mathrm{rpm}$

$8.609 \mathrm{~kg} / \mathrm{s}$

1.354

$90.65 \%$

$0.6096 \mathrm{~m}$

0.5

$2.448 \times 10^{5}$

0.843

0.438

0.509

0.570

0.780

1.500

$1.328 \mathrm{~mm}$

$0.667 \mathrm{~mm}$

$0.686 \mathrm{~mm}$ and a torque-meter. The plenum (in the shape of a scroll) provides axisymmetric uniform flow and temperature distributions at the inlet to the compressor. The flow path consists of a honeycomb section with screens to assure a uniform flow distribution at inlet. A contraction section is also provided to accelerate the flow smoothly into the IGV section of the compressor. A throttle located downstream of the compressor is used to control the mass flow through the compressor. The throttle system includes a surge control device, which activates a quick release feature of the discharge throttle, for rapid stall recovery in the event of surge. The surge control system features a differential pressure transducer which senses the pressure difference between the exit of the compressor and the exit plenum. If this pressure difference is higher than a previously set set-point, the throttle is opened for rapid recovery. The flow is exhausted through a duct into an exhaust anechoic chamber to reduce noise levels.

A schematic of the compressor flow path and measurement locations is shown in Figure 1. The outer annulus wall has a constant diameter of $0.6096 \mathrm{~m}$ (24.0 in). The hub wall diameter varies from $0.5075 \mathrm{~m}$ (19.98 in) at the inlet to $0.5232 \mathrm{~m}$ (20.6 in) at the exit. All the stators are cantilevered from the outer annulus wall with a rotating hub. Table 2 documents the mean line blading details. The average dynamic tip clearance is $1.38 \%$ for all the three rotors based on span of the rotor blade. This compressor is similar in geometrical, aerodynamic and performance characteristics to $\mathrm{S} 1$ and $\mathrm{S} 2$ compressors built by the Pratt and Whitney Compressor Group (Behlke et al., 
TABLE 2

Specifications of Test Compressor Blading

\begin{tabular}{|c|c|c|c|c|c|c|c|}
\hline Parameter & IGV & Rotor 1 & Stator 1 & Rotor 2 & Stator 2 & Rotor 3 & Stator 3 \\
\hline No. of Blades & 100 & 70 & 71 & 72 & 73 & 74 & 75 \\
\hline Dia. (m) & 0.5569 & 0.5587 & 0.5603 & 0.5618 & 0.5632 & 0.5644 & 0.5659 \\
\hline \multicolumn{8}{|l|}{ Blade inlet } \\
\hline mean camber angle (deg) & -6.40 & 52.97 & 46.48 & 53.72 & 48.91 & 52.45 & 47.01 \\
\hline \multicolumn{8}{|l|}{ Blade exit } \\
\hline mean camber angle (deg) & 26.60 & 27.50 & 14.53 & 27.38 & 16.49 & 27.59 & 21.33 \\
\hline Total camber angle (deg) & 33.10 & 25.47 & 31.95 & 26.34 & 32.42 & 24.86 & 25.68 \\
\hline Chord angle (deg) & 13.50 & -40.23 & 58.05 & -40.55 & 32.70 & -40.02 & 34.17 \\
\hline Chord (m) & 0.01829 & 0.03218 & 0.03175 & 0.03132 & 0.03089 & 0.03089 & 0.03048 \\
\hline Space-chord ratio & 0.955 & 0.779 & 0.781 & 0.783 & 0.785 & 0.776 & 0.778 \\
\hline Thickness to chord ratio & 0.060 & 0.065 & 0.085 & 0.065 & 0.085 & 0.065 & 0.085 \\
\hline Span (m) & 0.05253 & 0.05093 & 0.04928 & 0.04783 & 0.04643 & 0.04516 & 0.04382 \\
\hline \multicolumn{8}{|l|}{ Avg. Tip Clearance } \\
\hline $\begin{array}{l}\text { Static for rotor, } \\
\text { static for stator }\end{array}$ & - & $2.748 \%$ & $1.546 \%$ (Hub) & $2.751 \%$ & $1.477 \%(\mathrm{Hub})$ & $2.806 \%$ & $1.797 \%$ (Hub) \\
\hline Aspect ratio & 2.879 & 1.580 & 1.550 & 1.530 & 1.500 & 1.460 & 1.440 \\
\hline
\end{tabular}

All angles are measured with respect to axial direction, tip clearance is normalized by the blade span.

[1979]). All rotor and stator sections are NACA 65/CA airfoils (i.e., NACA Series 65 thickness distribution symmetrically distributed around a circular arc mean camber line). Minor modifications were made to the thickness distribution near the trailing edge to eliminate Series 65 hook. The inlet guide vane (IGV) is a NACA 400 series airfoil.

\section{INSTRUMENTATION AND DATA ACQUISITION}

Total pressure and total temperature measurements were carried out using seven element kiel total pressure and temperature pole rakes, respectively. Wall static pressures were distributed throughout the casing of the compressor in order to evaluate individual stage behaviour. Stator leading edge kiels were used to evaluate the variation in total pressure throughout the compressor.

Detailed spanwise flow field measurements at the inlet (station 1) were obtained using a miniature five hole probe of $1.67 \mathrm{~mm}$ head diameter at 31 radial locations and four circumferential locations. A single sensor hot-wire probe was radially traversed at one circumferential location at the inlet to record the mean and the turbulence data. The radial profile was discretized into 31 points in the same manner as that of the five hole probe measurements. The pressure measurements were carried out using a 64 channel measurement system with dynamic calibration, equipped with a pressure calibration unit which automatically calibrates all 64 channels at once using a Digiquartz reference pressure transducer. The temperatures were measured using an Omega Thermal Scanning System which consists of K-type thermocouples, a K-type CJ compensator and an signal amplifier. The pressure and temperature data were acquired using the Metrabyte DAS-20 data acquisition system. The data at stator 2 exit was acquired with a miniature five-hole probe of $1.07 \mathrm{~mm}$ dia.

\section{AREA TRAVERSE MECHANISM}

In order to acquire instantaneous pressure, velocity and turbulence data in an embedded stage, an area traverse mechanism was designed and built at Penn State. The area traverse mechanism shown in Figure 2 allows detailed area traverse of $1 \frac{1}{2}$ blade passages downstream of stator 2 (station 6), downstream of rotor 3 (station 7) and downstream of stator 3 (station 8). Four different

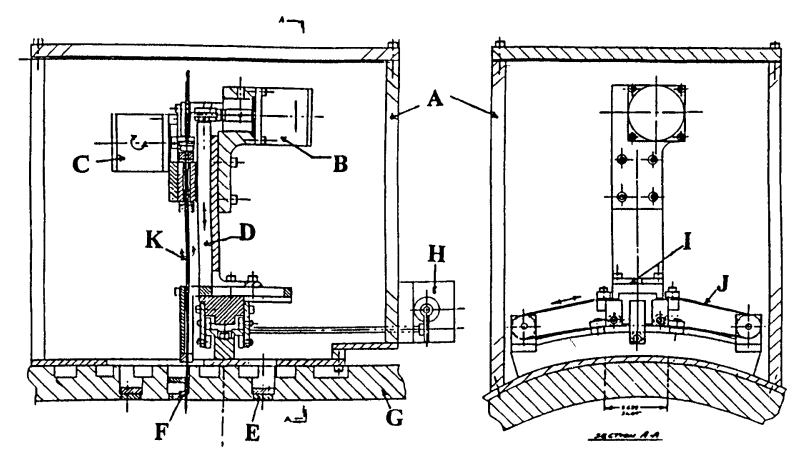

FIGURE 2 Schematic of Area Traverse Mechanism. 
types of probes (five-hole probe, aspirating probe, singlesensor slanted hot-wire and the thermocouple probe) can be traversed using the mechanism. The traverse mechanism allows for tangential and radial traverse and probe rotation about its axis ( 3 degrees of freedom). The mechanism is enclosed in a sealed chamber (A) which is mounted on the compressor outer casing. The probes $(\mathrm{K})$ are encased in a teflon shoe $(\mathrm{F})$ which is traversed in a slot $(\mathrm{E})$, which ensures a smooth interface between the probe and the casing wall $(\mathrm{G})$. The teflon shoes are designed to be at least three probe diameters wide on each side, to minimize local flow disturbances. The probes are traversed by stepper motors $(\mathrm{B}, \mathrm{C}$ and $\mathrm{H})$ driven by a computer. The stepper motors are of 0.10565 kg.m torque and are controlled by a stepper motor controller. The stepper motor for tangential traverse $(\mathrm{H})$ moves the chain driven sprocket which moves the traverse gear (I) using the chain (J). Radial motion is achieved by stepper motor (B) which moves the slide (D). Stepper motor $\mathrm{C}$ is utilized for rotating the probe about its own axis. Provisions are made for interfacing pneumatic and electrical lines through the side wall of chamber to provide both motion control and data acquisition. The area traverse mechanism can be driven by either a software driver interfaced with the software to acquire pressure data or a command file format to acquire data with the data acquisition system. The area traverse can be stepped in very fine increments; thereby very high accuracy can be maintained. The minimum step size is $0.000115 \mathrm{~mm}$ in the radial direction and $0.000454^{\circ}$ in the tangential direction. The positioning is accurate to $\pm 0.05 \mathrm{~mm}$ in radial and tangential positioning and $\pm 0.0225^{\circ}$ in probe rotational positioning.

\section{OVERALL PERFORMANCE AND INLET AND EXIT FLOW FIELD}

The overall performance characteristics were measured and documented at three different rotor operating speeds (100, 85 and $105 \%$ of the design corrected rotor speed) and at a set of operating throttle conditions. Details of the data reduction procedure and equations are given in Lakshminarayana et al. [1991]

The total pressure rise characteristics at the three corrected rotor speeds are shown in Figure 3(a). The pressure ratio increases with increase in rotor speed with the peak pressure ratio being 1.26 for $85 \%$ speed and 1.37 for $100 \%$ speed. For the $105 \%$ speed, the peak pressure ratio was not reached since the compressor input power and speed was limited. The torque based efficiency is shown in Figure 3(b). The performance of
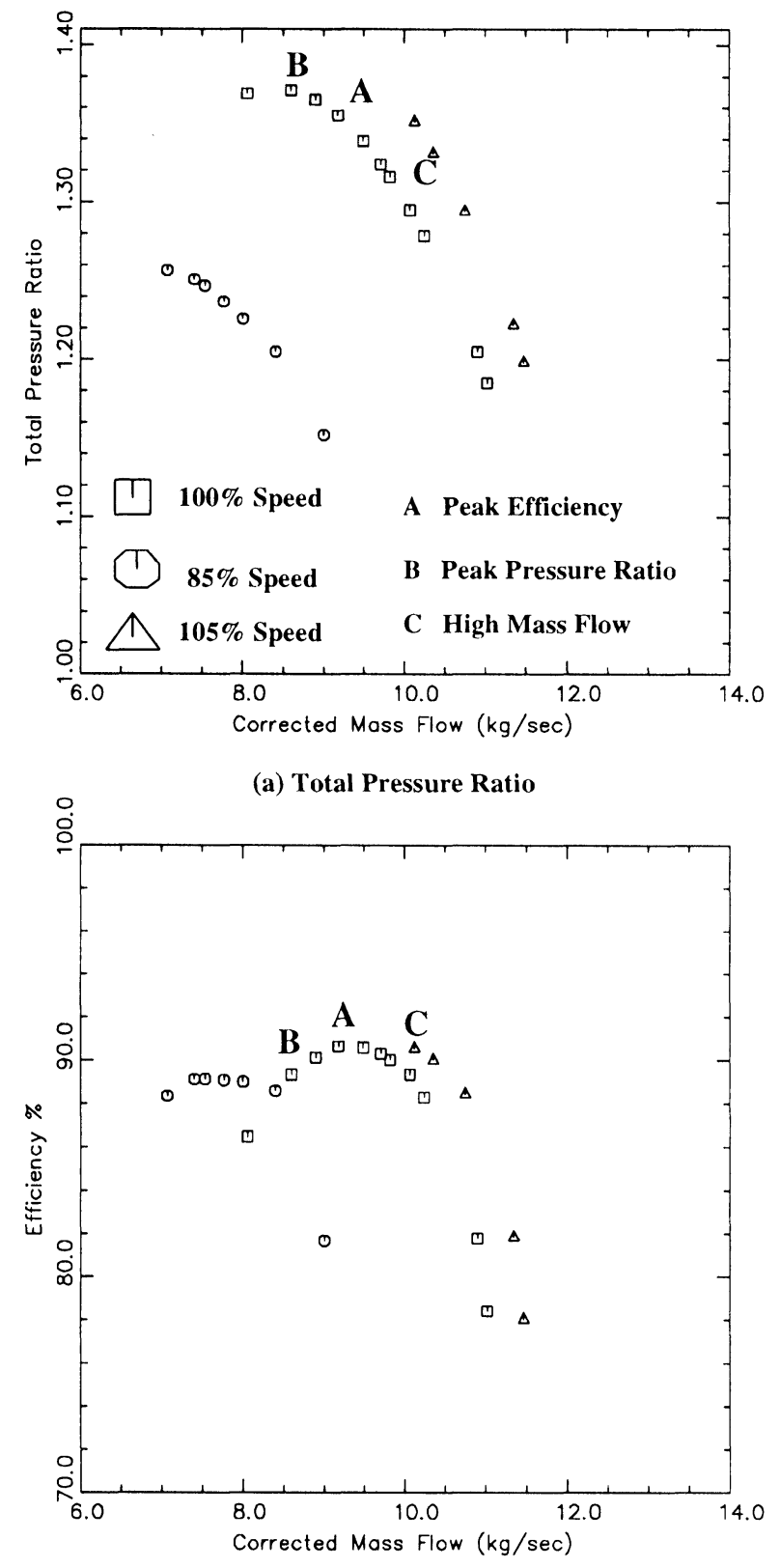

(b) Torque Efficiency

FIGURE 3 Performance Characteristics at Three Corrected Rotor Speeds.

the compressor is similar to the $\mathrm{P} \& \mathrm{~W}$ compressor $3 \mathrm{~S} 1$ which is of similar design (Behlke et al. [1979]). The design speed has the highest peak efficiency of $90.65 \%$ achieved at $100 \%$ speed. Hence, design performance has been achieved. All of the data reported and interpreted in this paper are taken at the peak efficiency point (A in Figures 3(a) and 3(b)). The casing static pressure data 
has been reported at 3 operating conditions (peak efficiency point $\mathrm{A}$, peak pressure ratio point $\mathrm{B}$ and high mass flow point C (Figures 3(a) and (b)).

In order to characterize the axisymmetry in the inlet flow field, data was acquired at the peak efficiency point at $100 \%$ corrected speed. The radial distribution of inlet velocity and pressures was derived from the five hole probe, as well as inlet total pressure and temperature rakes at several circumferential locations. The radial distribution of turbulence intensity and the deterministic unsteadiness at the inlet were measured by a single sensor hot-wire probe. The flow is found to be axisymmetric, except in the hub wall boundary layer regions. The annulus wall boundary layer is approximately $15 \%$ of the span and the hub wall boundary layer is approximately $10 \%$ of the span. The inlet tangential and radial velocities were found to be negligibly small at all circumferential locations across the span.

A single sensor hot-wire, placed normal to the axial flow direction is used to measure the axial and the radial component of total unsteadiness (including various turbulence) defined by

$$
T u=\frac{\sqrt{\overline{u^{\prime 2}}+\overline{w^{\prime 2}}}}{V}
$$

where $u^{\prime}$ and $w^{\prime}$ are the fluctuations in the axial and the radial direction. The overall unsteadiness varies from $3 \%$ at mid-span to $9 \%$ and $14 \%$ very near the hub and casing walls respectively. The variation of unsteadiness is almost negligible in the core region as expected.

Detailed radial traverses of the five hole probe at three different circumferential positions was conducted to investigate the nature of the exit flow field. Since the exit measurement station is 2.774 chords downstream of stator 3 , the flow is expected to be nearly axisymmetric at this location. Spanwise distribution of local total pressure ratio $\left(P_{r}\right)$, axial velocity, radial velocity and absolute flow angles at station 10 are shown in Figure 4. It is clear that the flow has not yet achieved axisymmetric conditions, even though radial profiles are similar at all three tangential measuring locations. The effects of blade wakes, secondary flow and leakage flow still persist. These distributions show that the core region is small (roughly one third of the blade height) and the endwall effects are substantial. The velocity distribution in the endwall region shows no noticeable presence of leakage flow/vortex from rotor 3. As indicated in Lakshminarayana et al. [1994], the mixing of the leakage flow with the viscous layers in the endwall region results in a complex distribution of velocity and pressure. It is thus clear that the rotor 3 tip clearance effects have been
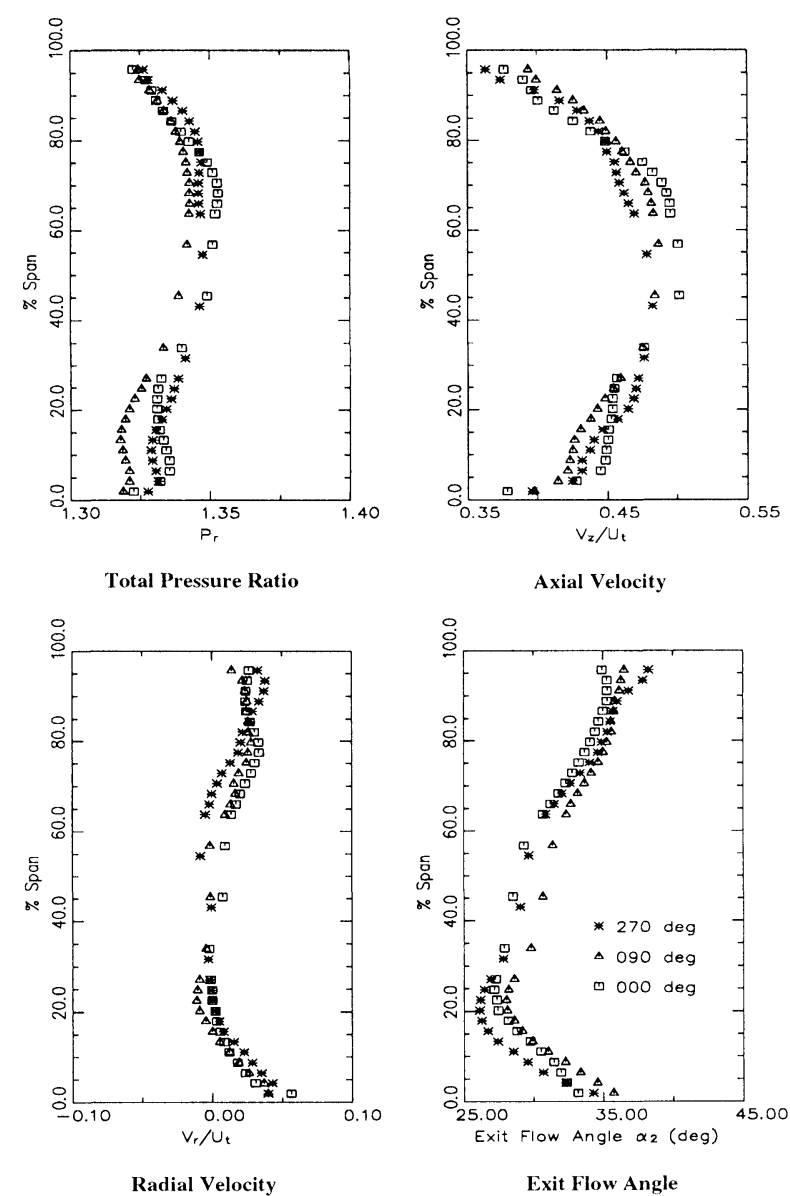

FIGURE 4 Flow Distribution at Exit: Five hole probe data (peak efficiency condition, $100 \%$ speed).

mixed out, while those due to stator 3 hub clearance still persist at this location. The hub region (up to $20 \%$ of span) is characterized by low axial velocity, low stagnation pressure rise, large underturning (the mean line stator 3 blade exit angle is $21.33^{\circ}$ ). The rotor 3 hub wall separation, reported later, may also contribute to low axial velocities in this region.

\section{PERFORMANCE OF EMBEDDED STAGES}

The evaluation of casing pressures will be useful in determining whether the flow behaviour is as expected inside the stages. The casing static pressure distribution along the compressor will enable the detection of stall or abnormal behavior in the flow field. The casing pressure data was acquired simultaneously with the performance data at the same operating points. The details of the casing static pressure distribution are given in Lakshminarayana et al. [1991]. 
The radial distribution of flow properties is based on mass average ( 31 points) across the pitch of stator 2 ; at inlet, exit of stator 3 and downstream of the compressor the circumferential average is based on several (typically 4-5) tangential locations as indicated.

\section{Total Pressure}

The spanwise variation of averaged local total pressure ratio $\left(P_{r}\right)$ measured at various locations along the compressor using the five hole probe is shown in Figure 5, at the peak efficiency point at $100 \%$ speed. The five hole probe data downstream of stator 2 has been mass averaged across one blade passage whereas the five hole probe data at inlet, exit and downstream of stator 3 has been circumferentially averaged. The flow is very well
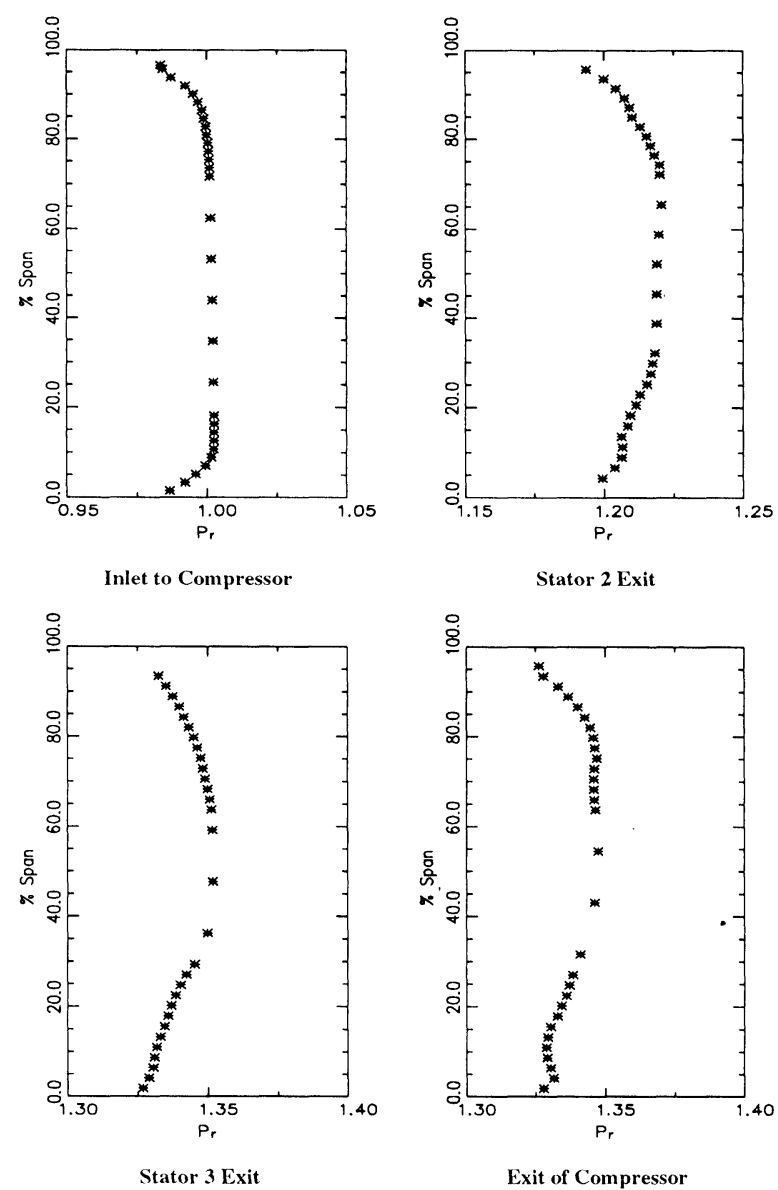

FIGURE 5 Spanwise Variation of Total Pressure Profiles Across the Compressor at the Peak Efficiency Operating Condition, 100\% speed. behaved at the inlet and through stage 1 . The tip region at the exit of stators 2 and 3 has a conventional profile with monotonic increase in pressure from the endwall to freestream. The hub region, however, has a complex profile due to interaction of hub wall boundary layer and the leakage of the flow due to the cantilevered stator blade. Similar characteristics have been measured by Dransfield and Calvert [1976], Calvert et al. [1989] and Falchetti [1992]. There is no deterioration of the flow as it passes through the compressor except for increased viscous layers in the endwall region. The flow downstream of the third stator is very similar to the flow downstream of the second stator.

The core flow region, which is approximately $75 \%$ of the span at inlet, reduces gradually to less than $30 \%$ of span at the exit of the compressor. Since the measurements shown in Figure 5 are at the exit of stators, the leakage effects due to rotor tip clearances are not observed. The data indicate that the leakage flow has mixed within the stator passage, resulting in a conventional viscous layer (monotonically decreasing towards the annulus wall). Whereas the flow near the hub shows the effect of leakage due to clearance between a stationary blade and a rotating hub, the values of Cpt increase from hub to about $10 \%$ of span, followed by a flat profile (and a wake type of region at exit). This is typical of profiles caused by the interaction of leakage flow with the primary flow (Lakshminarayana et al. [1995]).

\section{Flow Angle and Axial Velocity}

The absolute flow angle at the exit of stators 2 and 3 and at the exit of the compressor are shown in Figure 6. The inlet flow was axial. The design angles are also shown in this figure. Downstream of stator 2, beyond $70 \%$ span, the measured profiles show under-turning up to about $95 \%$ span. Over-turning is observed in the outer $5 \%$ of the stator blade span, caused by secondary flow. Both underturning and overturning are observed near the hub. Up to about $9 \%$ of the span from the hub, the flow is underturned. The overturning is confined to spanwise locations extending from $10 \%$ to $25 \%$ span. This is typical of the distribution observed in vortical motion. This may also have been caused by separated flow in the upstream rotor, (as explained earlier), and the associated shear gradient, resulting in secondary flow and overturning in this region. Downstream of stator 3, the radial distribution of exit flow angle is very similar to those observed at the exit of stator 2 . The underturning region near the hub has increased. The passage averaged pitch angle distribution were found to be small at all axial 


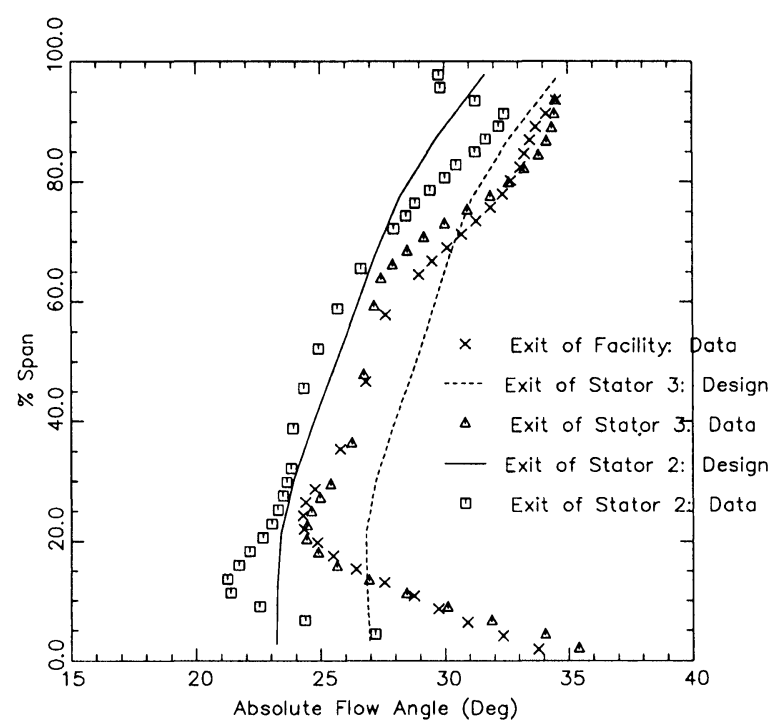

FIGURE 6 Spanwise Variation of Absolute Flow Angle Across the Compressor at the Peak Efficiency Operating Condition, 100\% speed.

locations and were of the same order of magnitude as the measurement uncertainties.

The spanwise variation of average axial velocity at several axial locations along the compressor at the peak efficiency point, $100 \%$ speed is shown in Figure 7. The mass flow based on measured local axial velocity is found to be almost the same at all axial locations. The flow is uniform in the mid-span regions with variations occurring in the end-wall regions due to the effect of various flow mechanisms (hub clearance flow from stators, tip clearance flow from the rotors, annulus wall boundary layers and secondary flow).

The radial distribution of average axial velocity indicates substantial changes as the flow goes through several blade rows. The boundary layer thickness at the inlet is approximately $10 \%$ near the hub and $10-15 \%$ near the tip. This grows substantially as the flow progresses through the compressor. The hub wall flows show the same trend as Cpt where the stator-hub leakage flow interacts with the main flow to produce unconventional boundary layer-wake type of profiles near the hub wall region. This may also have been caused by a scraping vortex and the upstream rotor hub separated flow. This region extends from $10 \%$ to $20 \%$ of the span from the hub.

The leakage flow tends to reduce the boundary layer growth, especially very near the wall. This is clearly seen from a comparison of the velocity profiles near the hub and the tip. The flat portion of the profile (from 10 to
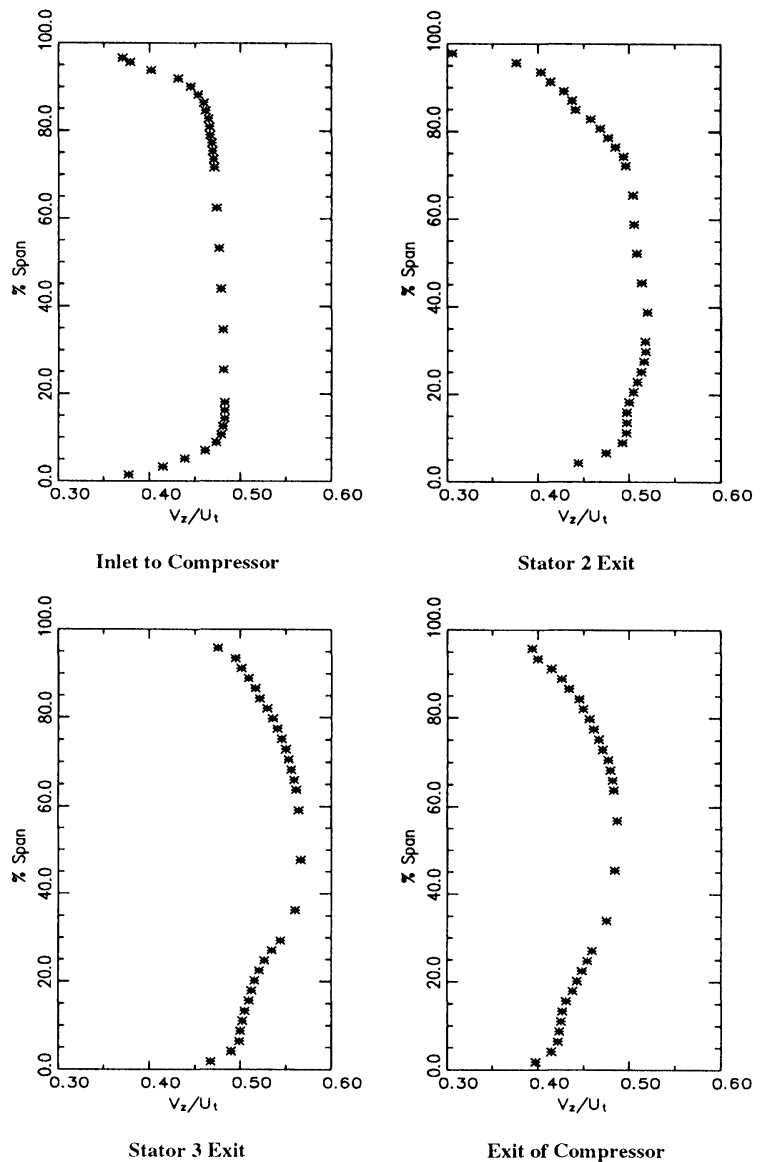

FIGURE 7 Spanwise Variation of Absolute Axial Velocity Across the Compressor at the Peak Efficiency Operating Condition, 100\% speed.

$20 \%$ span), which has higher than expected values (of the extrapolated boundary layer profile from 30 to $20 \%$ ) may have been caused by either a scraping vortex or the upstream separated flow in the rotor. The separated flow at the exit of the rotor has higher absolute velocity than the un-separated flow. This hypothesis is supported by higher than expected temperature rise (see Figure 8) in this region. This hypothesis is supported by the fact that the static pressure is nearly invariant across the span at this location.

\section{Total Temperature}

Detailed area traverses were conducted using a low frequency response thermocouple at $5.6 \%$ chord downstream of stator 2 trailing edge. The circumferentially area averaged total temperature ratios (based on mass averaged inlet total temperature) are plotted in Figure 8 for the peak efficiency condition. Also shown on these 


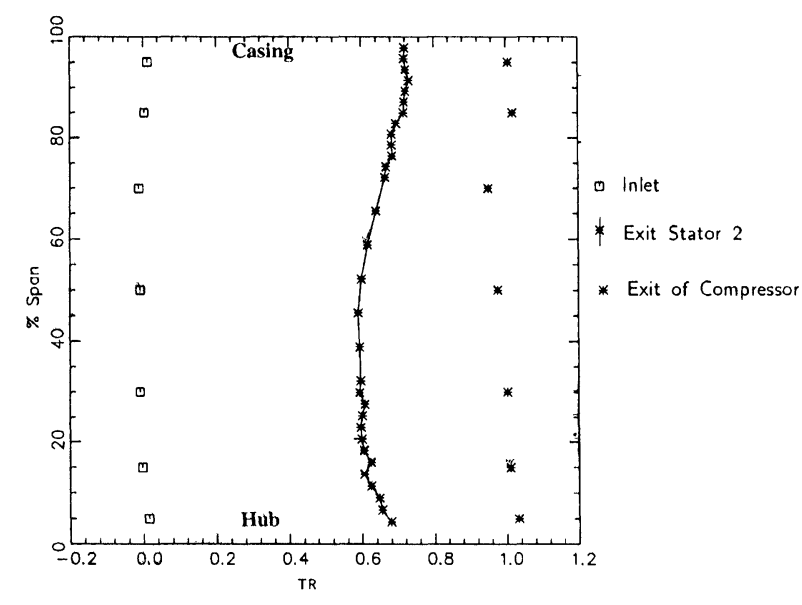

FIGURE 8 Spanwise Variation of Total Temperature Rise Coefficient Across the Compressor at the Peak Efficiency Operating Condition; $100 \%$ speed.

plots are the circumferentially averaged radial distributions of inlet and exit total temperature ratio. The temperature field is very well behaved at the inlet where the temperature distribution is almost uniform in the radial direction. Downstream of stator 2 the profiles already show evidence of higher temperatures at the end walls with the temperature near the tip higher than that at the hub. Similar behavior is reported by Falchetti [1992]. Higher stagnation temperatures near the endwalls are caused by viscous layers and the associated higher temperature rise across the rotors in these regions. At the compressor exit, the radial gradients in total temperature near the endwalls are much lower than the radial gradients at corresponding spanwise locations downstream of stator 2. This is due to increased spanwise mixing of the flow downstream of stator 3. Similar distributions were measured by Behlke et al. [1979] and computed by Adkins and Smith [1982]. Calculations to predict the total temperatures at the exit of stators without mixing by Adkins and Smith [1982] have shown high total temperature at the endwalls compared to the midspan region.

\section{Isentropic Efficiency}

Radial distributions of isentropic efficiency of the first two stages calculated downstream of stator 2 (based on the total pressure and total temperature traverses at this location) is shown in Figure 9. Isentropic efficiency $\left(\eta_{\text {isen }}\right)$ is defined by:

$$
\eta_{\text {isen }}=\frac{P_{r}^{\left(\frac{\gamma-1}{\gamma}\right)}-1}{T_{r}-1}
$$

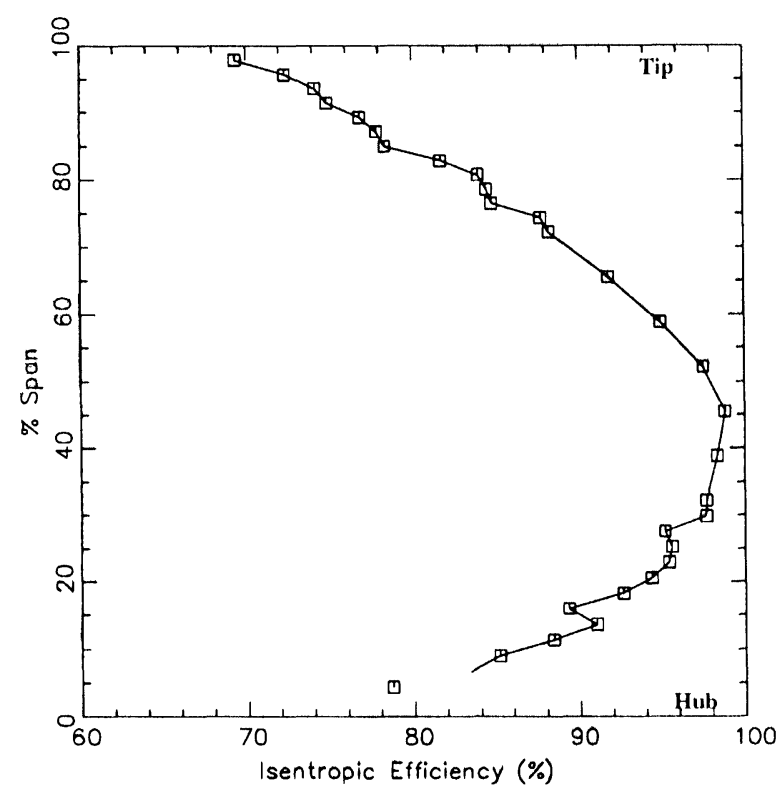

FIGURE 9 Spanwise Variation of Isentropic Efficiency Across the Compressor at the Peak Efficiency Operating Condition, $100 \%$ speed.

Measured isentropic efficiency downstream of stator 2 is the lowest at the hub and tip regions and highest in the midspan regions as is expected: With higher losses in the casing regions (lower total pressure ratios and higher total temperature ratios), the efficiencies are much lower than the efficiencies in the hub regions. The tip regions have the lowest efficiency indicating that the rotor leakage flow and the stator annulus wall secondary flow are more severe than their counterpart near the hub. Similar distributions have been measured by Calvert et al. [1989].

\section{FLOW FIELD AT THE EXIT OF STATOR 2 (AREA TRAVERSE)}

A complete area traverse was performed at the exit of stator 2 to resolve the detailed time average flow field properties, including three mean velocity components and total and static pressures. A sub-miniature $(1.07 \mathrm{~mm}$ tip) five-hole probe was traversed 5.6\% stator 2 chord (at mid-span) downstream of stator 2 (Figure 1). The area traverse data was obtained at the peak efficiency condition (point $A$ in Figure $3(a)$ ) and a $31 \times 31$ grid (with grid point clustering in the wake and endwall regions) was utilized to finely resolve the flow field. The tangential traverse was $188 \%$ of one stator 2 pitch at mid-span. A total pressure kiel probe is located at $90.1 \%$ span on the 
leading edge of the stator blade at $100 \%$ stator pitch. The blade with the leading edge kiel probe will be referred to as the "kiel" blade, whilst the blade with no kiel probe will be referred to as the "clean" blade.

Figure 10 shows the total pressure coefficient contours at the peak efficiency operating condition. The effects of secondary flow and thickening of the boundary layer near the suction surface corner in the casing endwall region is clearly seen. The "inviscid" core region extends from approximately $20 \%$ span from the hub to $80 \%$ of the span. The wakes in this region are approximately $20 \%$ of the blade spacing. Lowest total pressures are observed in the wakes in the endwall region with $\mathrm{Cpt}$ values as low as 4.5. The wakes are much thicker at the casing in the outer $20 \%$ of span for the "clean" blade and $30 \%$ of the span for the blade with leading edge kiel. The total velocity profiles presented later indicate no separated flow region at the corner formed by the suction surface and the casing at this peak efficiency condition.

The stagnation pressure contours near the hub indicate a low energy core located near the mid-passage, possibly caused by the leakage flow in the hub/stator clearance region. The leakage and core region extends to nearly $10 \%$ of the span. Under-turning in this region, reported later, is expected as the blades are unloaded near the hub endwall. No measurements were taken closer than $2 \mathrm{~mm}$ from the rotating hub, hence no definitive conclusion can be drawn with regard to the presence of leakage or secondary vortex. The endwall boundary layer thickness in this region is larger than the tip gap, hence the presence of scraping is expected.

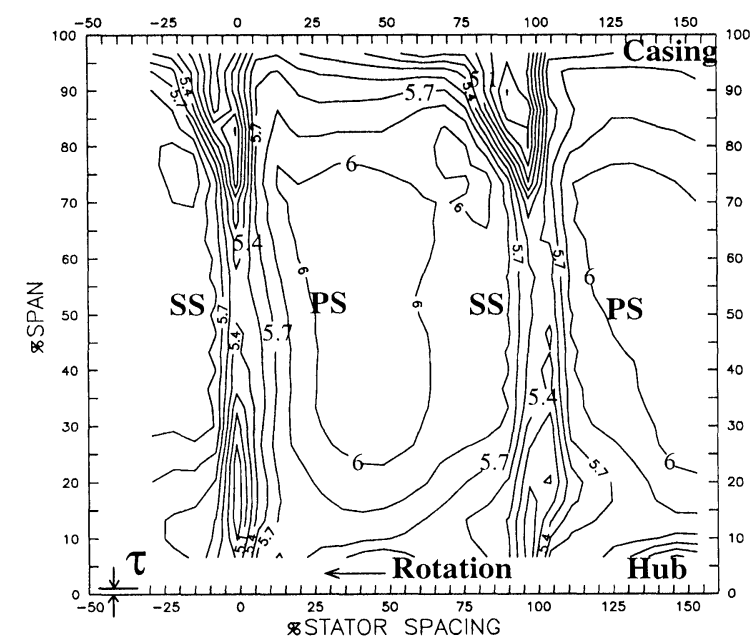

FIGURE 10 Total Pressure $\left(C_{p t}\right)$ Contours Downstream of Stator 2: Peak $\eta$; Interval $=0.15, \min =4.5, \max =6.1$.
Figure 11 shows the total velocity contours at the peak efficiency operating point. A large increase in the stator wake width in the casing endwall region on the suction side of the stator, caused by secondary and corner phenomena, can be clearly seen. The spanwise distance (h) of the loss core from the endwall, non-dimensionalized by the stator 2 mid-span chord, is $h / c=14.8 \%$ (or $90 \%$ span) for the left ("clean") blade wake and $h / c=$ $19.2 \%$ (or $87 \%$ span) for the right ("kiel") blade wake. The wake thickness near the rotating hub is not as significantly affected as in the casing endwall region as the stator hub clearance unloads the stator blade at the hub. The suction surface corner near the casing has low velocities at the peak efficiency point, but the axial velocity contours (not shown) indicate that the flow is not separated near the casing at this operating condition. The hub region reveals no separated regions. Even in the leakage vortex/mixing region, the axial velocities are higher than that encountered in the wakes.

The swirl angle contours are shown in Figure 12, for the peak efficiency operating conditions. The design exit angle for stator 2 varies from $23.2^{\circ}$ at the root to $31.63^{\circ}$ at the tip. The swirl angle distribution across the wake indicates the usual overturning and underturning on the suction and pressure side of the wakes, respectively. This is consistent with the wake data in a single stage compressor rotor (Prato and Lakshminarayana, 1993). The endwall region has overturning in the corner region formed by the suction surface and the wall and this is caused by the secondary flow. The flow on the pressure side in this region is underturned.

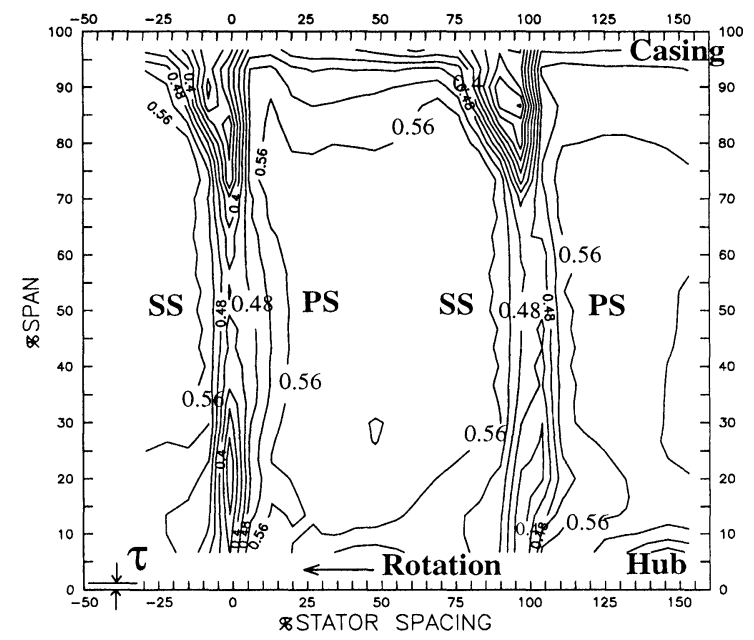

FIGURE 11 Total Velocity $\left(V_{t o t} / U_{t}\right)$ Contours Downstream of Stator 2: Peak $\eta$; Interval $=0.04, \min =0.16, \max =0.60$. 


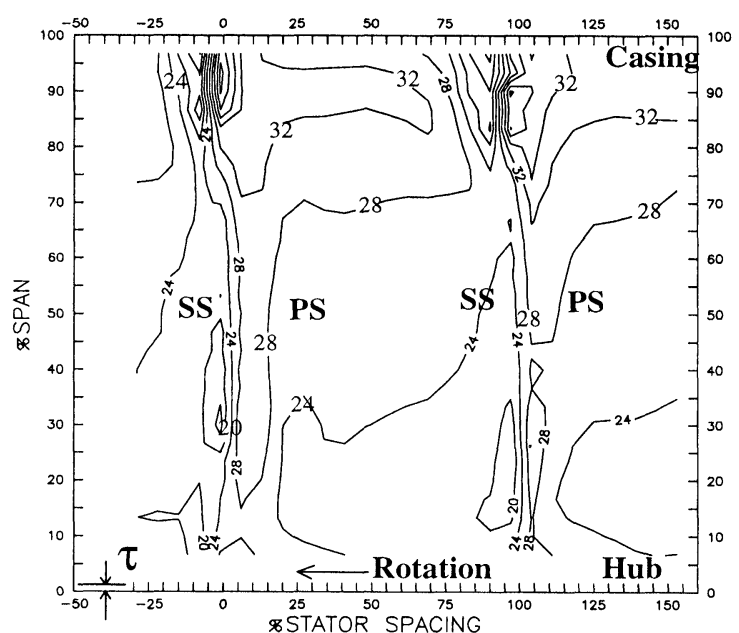

FIGURE 12 Swirl Angle (in degrees) Contours Downstream of Stator 2: Peak $\eta$; Interval $=4.0, \min =12.0, \max =48.0$.

Figure 13 shows the secondary flow vector plots for the peak efficiency operating condition. The secondary flow vectors are defined as the vector sum of the radial velocity component and the difference between the measured and the design tangential velocity. All the radial flow, therefore, is considered a component of the secondary flow, whereas the tangential component of the secondary flow is defined relative to the exit "design" angle. Therefore, the tangential component (in the bladeto-blade direction) must be viewed with some caution since it is defined relative to an exit flow angle distribu-

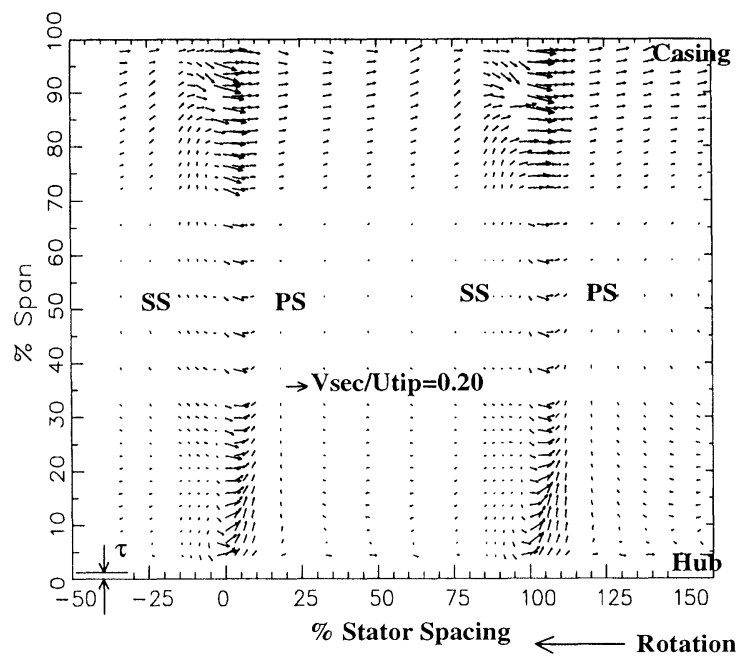

FIGURE 13 Secondary Flow Vectors Downstream of Stator 2: Peak $\eta$. tion which may or may not represent the true primary flow direction at this location.

The secondary flow is nearly zero in the mid-passage at mid-span. In the casing endwall region, the secondary flow moves from the pressure side to the suction side. The fluid near the suction side is directed towards the center of the low total pressure region earlier identified to have a core at $90 \%$ span for the left or "clean" blade. As indicated by the vectors, the core region entrains fluid from above (inward flow) and below (outward flow) the low energy core. This is illustrated by the direction of the secondary velocity vectors at this location. This migration of fluid is more pronounced for the right or "kiel" blade (Figure 13) as the local fluid is entrained towards the center of the low total pressure core earlier identified as occurring at $87 \%$ span.

The flow in the wakes from $30 \%$ to $70 \%$ span is radially inward due to the local imbalance between the centrifugal forces acting on the fluid particles and the radial static pressure gradient in the stator blade wakes. The secondary flow vectors from $5 \%$ to $20 \%$ span are reflective of the viscous effect of the stator blades scraping the skewed hub endwall boundary layer. The fluid near the pressure side of either blade is directed radially outward since the scraping action of the stator blade relative to the rotating hub causes the fluid to roll resulting in a clockwise vortex with radial outward flow on the pressure side. The viscous nature of the fluid causes the fluid on the suction side to be entrained inward and across the wake towards the pressure side of the blade. The blade-to-blade profiles of pitch angle (not shown) indicate radially inward flow on the suction side and radially outward flow on the pressure side of the stator blade wakes. The interpretation of the flow near the hub region is speculative and additional data near the clearance region is necessary before the phenomena can be attributed to the leakage or the scraping vortex.

\section{CONCLUSIONS}

The following conclusions can be drawn on the behaviour of the flow in multistage axial flow compressors based on the experimental investigation described in this paper.

1. The contour plots of various properties at the exit of stator 2 reveal that the blade wakes are about $20 \%$ of the blade spacing away from the endwalls at the peak efficiency operating conditions, and the wake width is as large as $35 \%$ of the spacing near the endwalls. Near the rotating hub, a region where the boundary layer scraping effect is present manifests itself in a 
loss core with large underturning. The flow downstream of stator 2 is non-periodic and non-uniform, especially in the hub and tip regions.

2. Absolute flow angle distributions downstream of stators 2 and 3 show the presence of underturned regions at both the hub and the tip regions, with constant angle core flow regions.

3. Secondary flow vectors measured downstream of stator 2 show the migration of the fluid particles toward the core of the low pressure regions located near the suction side of the casing endwall corner.

4. At the peak efficiency operating condition, the passage averaged quantities indicate that the viscous regions penetrate to nearly 30 to $35 \%$ of the span from both the casing and the hub endwall regions. Therefore, only $30 \%$ of the span remains inviscid "core" flow at this axial station within the machine.

5. The passage averaged radial velocity distributions show that the overall magnitude of the radial flows are small. Furthermore, the radial velocity distributions show that the radial velocities are nearly zero in the core region with deviations occurring predominantly near the endwalls and in the wakes.

6. The annulus wall regions have the lowest efficiency indicating that the rotor leakage flow and the stator annulus wall secondary flow are more severe than their counterpart near the hub.

\section{Acknowledgements}

The authors wish to acknowledge United Technologies Research Center (UTRC)/Pratt and Whitney (P\&W) Aircraft Division of United Technologies Corporation for donating the multistage compressor facility and to $\mathrm{H}$. Weingold, E. Canal and M. Christianson of UTRC/P\&W Aircraft for providing valuable advice and help in installing such a facility, in shakedown testing and in the performance evaluation. In addition, the following sponsors provided invaluable advice and funding for research; M. Howard of Rolls Royce, R. Fagan, P. Tramm and R. Delaney of Allison Engine Company, A. Strazisar of NASA Lewis Research Center. Installation of such a major research facility could not have been completed without funding from The Pennsylvania State University and help from many individuals notably; C. Camci, G. Sayers, H. Houtz, B. Dillon, J. Fetterolf, G. Gurney, R. Auhl, G. Fields and J. D. Miller.

\section{Nomenclature}

$C_{p t}$

$D S 2, D S 3$

$P_{o}$

$\bar{P}_{o}$

$\bar{P}_{o 1}$

$P_{R}$

$P_{r}$

PS

$P_{s}$

$\bar{P}_{s 1}$

$S$

$S S$

$T_{o}$

$\bar{T}_{o 1}$

$\bar{T}_{o 10}$

$T R$

$U_{t}$

$V_{t o t,}$,

$\alpha$

$\tau$

Subscripts:

$r, \theta, z$

1,2

Superscripts:

Denotes area/mass/time average

\section{References}

Adkins, G. G., Jr., and Smith, L. H., Jr., 1982, "Spanwise Mixing in Axial Flow Turbomachines," ASME Journal of Engineering for Power, Vol. 104, pp. 97-110.

Behlke, R. F., Bursdall, E. A., Canal, E. Jr., and Korm, N. D., 1979 , "Core Compressor Exit Stage Study: Part II-Final Report," NASA CR-159812.

Calvert, W. J., Ginder, R. B., McKenzie, I. R., and Way, D. J., 1989, "Performance of a Highly-Loaded HP Compressor," ASME Paper No. 89-GT-24.

Cherrett, M. A., and Bryce, J. D., 1992, "Unsteady Viscous Flow in a High Speed Core Compressor," ASME Journal of Turbomachinery, Vol. 114, pp. 287-294.

Dransfield, D. C., and Calvert, W. J., 1976, "Detailed Flow Measurements in a Four Stage Axial Compressor," ASME Paper No. 76-GT-46.

Total Pressure Coefficient $C_{p t}=\frac{\left(P_{o}-\bar{P}_{s 1}\right)}{\left(\bar{P}_{o 1}-\bar{P}_{s 1}\right)}$ respectively

Total pressure

Mass Averaged inlet $P_{o}$

Mass Averaged exit $P_{o}$

Total Pressure Ratio $\left(\frac{\bar{P}_{o 10}}{\bar{P}_{o 1}}\right)$

Local Total Pressure Ratio $P_{r}=\left(\frac{P_{o}}{\bar{P}_{o 1}}\right)$

Static pressur

Average inlet casing $P$

Total temperature of flow

inlet $T_{0}$

Total Temperature Rise Coefficient $T R=$ $\frac{\left(T_{o}-\overline{T_{o 1}}\right)}{\left(\overline{T_{o 10}}-\overline{T_{o 1}}\right)}$

Rotor blade tip speed

Absolute Total velocity

Flow angle measured from the axial direction

Clearance height (rotor tip or stator hub)

components

Inlet and Exit of each blade row 
Falchetti, F., 1992, "Advanced CFD Simulation and Testing of Blading in the Multistage Environment," AIAA Paper No. 92-3040.

Lakshminarayana, B., Suryavamshi, N., and Prato, J., 1991, "Experimental Investigation of Spanwise Mixing Phenomena and 3D Unsteady Flow in Multistage Compressors," PSU Turbo 9107, The Pennsylvania State University.

Lakshminarayana, B., Zaccaria, M., Marathe, B., 1995, "Structure of Tip Clearance Flow in Axial Compressors," ASME Journal of Turbomachinery, Vol. 115, p. 336.

Prato, J. P., and Lakshminarayana, B., 1993, "Investigation of the Compressor Rotor Wake Structure at Peak Pressure Rise Coefficien and the Effects of Loading," ASME Journal of Turbomachinery, Vol. 115, pp. 487-500.

Robinson, C. J., 1992, "Endwall Flows and Blading Design for Axial Flow Compressors," Axial Flow Compressors, von Karman Institute for Fluid Dynamics Lecture Series 1992-02 (Also Ph.D Thesis, Cranfield Institute of Technology, May 1991).

Stauter, R. C., Dring, R. P., and Carta, F. O., 1991, "Temporally and Spatially Resolved Flow in a Two-Stage Axial Compressor Part 1-Experiment," ASME Journal of Turbomachinery, Vol. 113, pp. 219-226. 

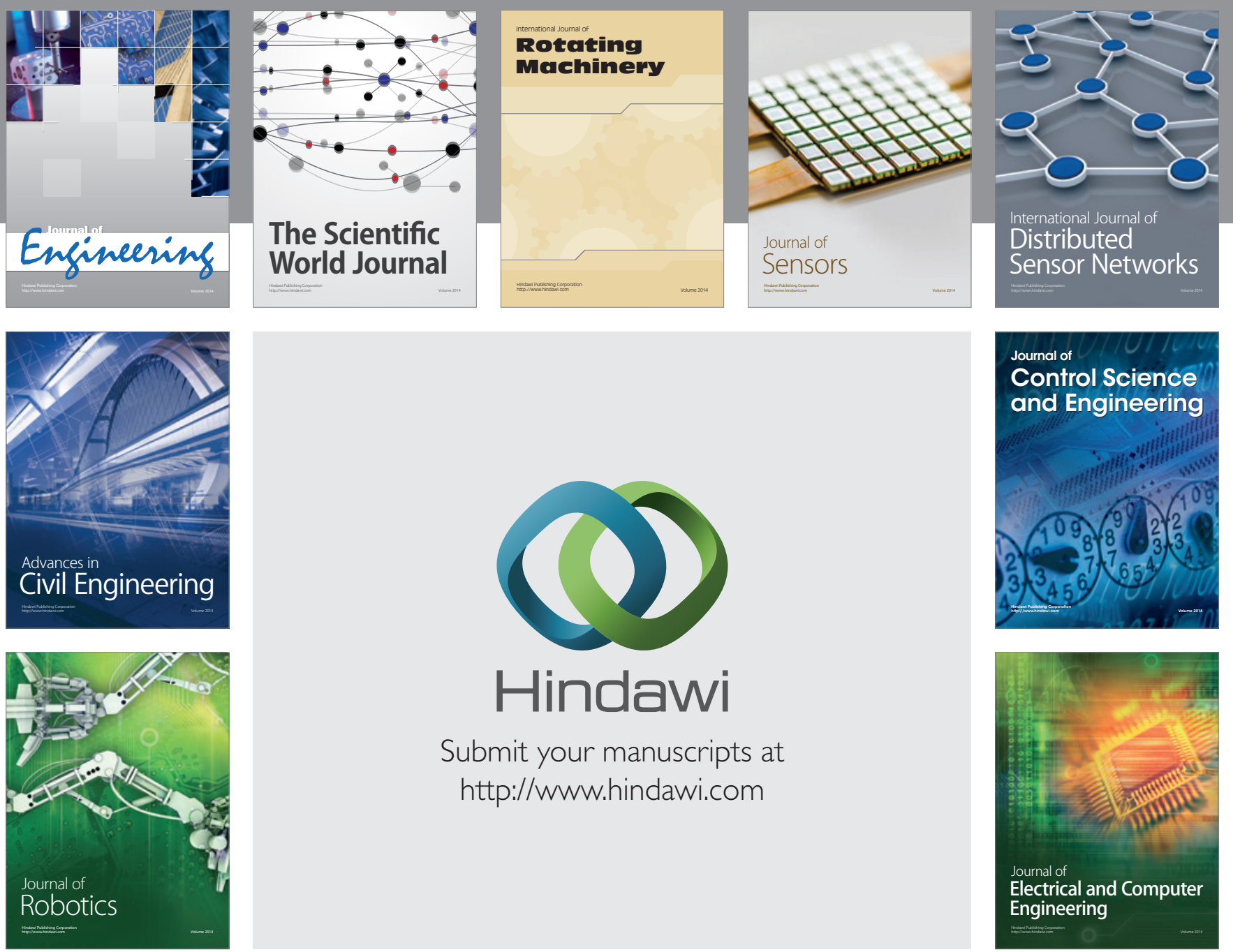

Submit your manuscripts at

http://www.hindawi.com
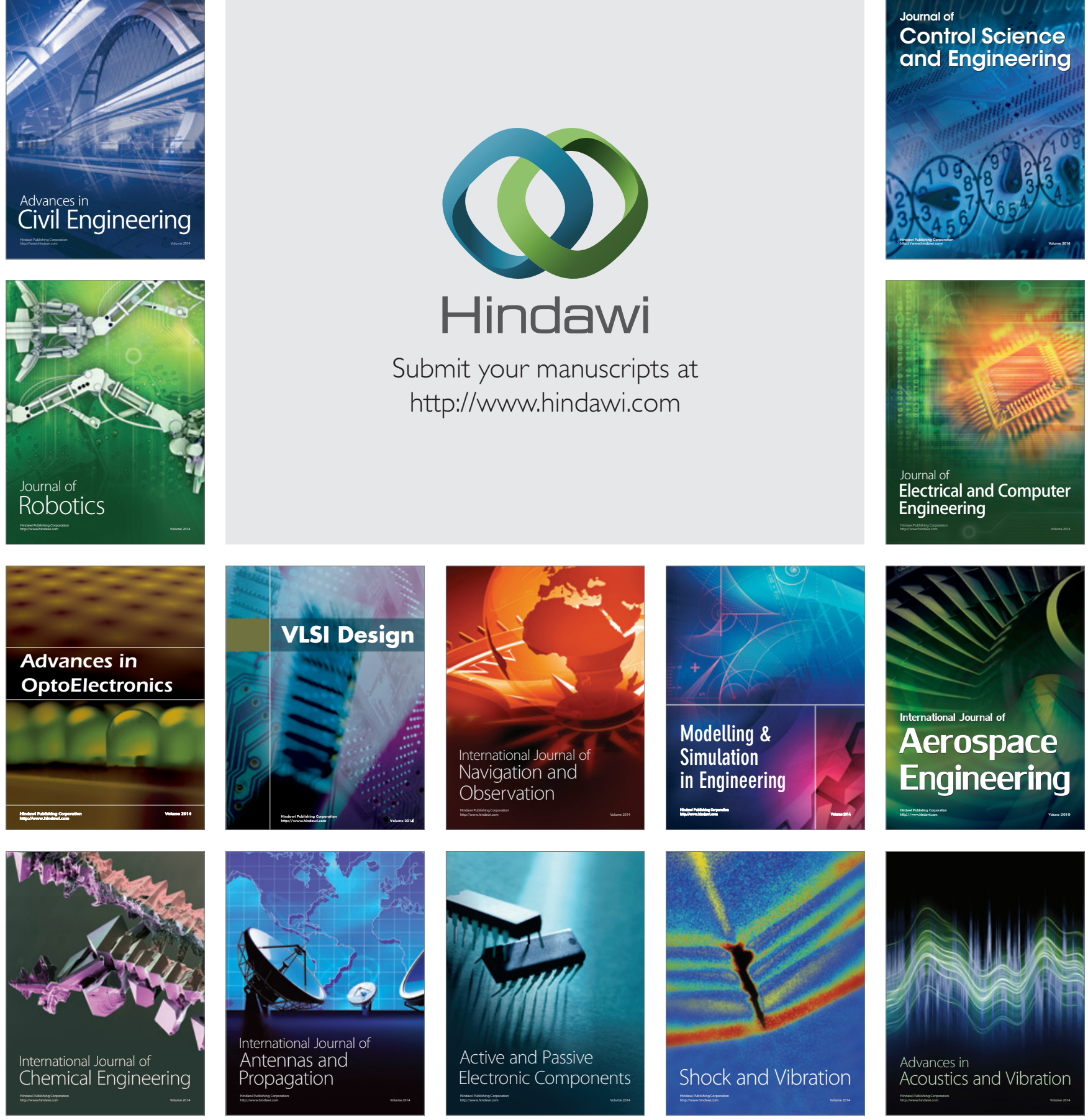